\title{
LA FORMACIÓN PEDAGÓGICA DE LOS PROFESORES UNIVERSITARIOS. UNA PROPUESTA EN EL PROCESO DE PROFESIONALIZACIÓN DEL DOCENTE
}

Maritza Cáceres Mesa y otros Universidad de Cienfuegos, Cuba

\section{INTRODUCCIÓN}

La Educación Superior se enfrenta a una serie de desafíos en un mundo que se transforma, por ello debe revisar su misión y redefinir muchas de sus tareas sustantivas, en especial aquellas que se relacionen con las necesidades de la sociedad en materia de aprendizaje y superación continua. Un punto clave en su misión está dirigido a destacar las tareas de las universidades, Se requiere llevar a cabo esfuerzos para elevar la formación pedagógica de los profesores, lo cual tributará en una mejor preparación de los egresados universitarios.

La actividad del profesor ha sido y seguirá siendo un aspecto de estudio de la Didáctica cada vez es más evidente su papel de facilitador en la calidad del proceso de enseñanza aprendizaje y en la educación en general.

La mejora de la calidad del proceso de enseñanza aprendizaje pasa necesariamente por la transformación del pensamiento y de los sentimientos de los profesores, para ello la Educación Superior necesita de la calidad del personal docente, de los programas y de los estudiantes, de las infraestructuras y del ambiente universitario.

Para que la Universidad pueda cumplir sus tareas académicas, laborales e investigativas requiere de profesores preparados, que no sólo sepan el contenido científico, sino que sepan enseñar lo que necesita la sociedad, de aquí la necesidad de que en la universidad se enseñe a los profesores a educar, para que los estudiantes aprendan a aprender.

En la Conferencia mundial sobre la Educación Superior de la UNESCO, se aprobaron documentos que insisten en la necesidad de la educación permanente del profesorado universitario y su formación pedagógica. En uno de esos documentos se especifica: "Un elemento esencial para las instituciones de enseñanza superior es una enérgica política de formación del personal. Se deberían establecer directrices claras sobre los docentes de la educación superior, que deberían ocuparse sobre todo, hoy en día, de enseñar a su alumnos a aprender y a tomar iniciativas, y no a ser, únicamente, pozos de ciencia. Deberían tomarse medidas adecuadas en materia de investigación, así como de actualización y mejora de sus competencias pedagógicas mediante programas adecuados de formación del personal, que estimulen la innovación permanente de los planes de estudio y los métodos de enseñanza aprendizaje, y que aseguren condiciones profesionales y financieras apropiadas a los docentes a fin de garantizar la excelencia de la investigación y la enseñanza". (1) 
Por otra parte en otro de los documentos se afirma: "Como la educación a lo largo de toda la vida exige que el personal docente actualice y mejore sus capacidades didácticas y sus métodos de enseñanza ... es necesario establecer estructuras, mecanismos y programas adecuados de formación del personal docente" (2)

Las contundentes declaraciones internacionales anteriores y las condiciones actuales del desarrollo de la educación superior cubana muestran una realidad que exige perfeccionar la formación pedagógica de los profesores universitarios en atención a las necesidades de aprendizaje de los mismos.

En el presente, resulta imprescindible la reflexión cotidiana sobre la tarea de enseñar y sus implicaciones pedagógicas según sus finalidades y contextos diversos.

La formación científica en la rama del saber específico debe ir acompañada de una formación pedagógica, sólo así puede incidir en el mejoramiento de su labor profesional.

La idea de la educación durante toda la vida, de la educación permanente es aplicable al profesional de la educación superior, pues el profesor universitario, en atención a las tareas docentes que realiza, requiere que disponga de posibilidades para su formación continuada.

\section{DESARROLLO}

La conceptualización de profesión asociada al proceso de enseñanza hace hablar de diversos estadíos de profesionalización reflejado en la clasificación de Mitchell y Kerchner (1983) y retomados por Imbernón (1994) quienes reconocen:

?? "El profesor como trabajador: Concibe la escuela como un sistema jerárquico del cual es gerente o director quién dice qué, cuándo y cómo debe enseñar el profesor, así las tareas de concepción y planificación están separadas de la ejecución.

?? El profesor como artesano. Se atribuye una mayor responsabilidad al docente para seleccionar y aplicar las estrategias de enseñanzas. En los programas formativos se prioriza la adquisición de trucos del oficio por encima de la teoría y la reflexión.

?? El profesor como artista. Se enfatiza la creatividad personal, y se permite el desarrollo de un mayor grado de autonomía docente. La adquisición de la cultura general y profesional está condicionada y tamizada por la institución, personalidad y dinamismo individual.

?? El profesor como profesional. El trabajo profesional por naturaleza no es propenso a la mecanización. El docente está comprometido con la autoreflexión y el análisis de las necesidades del alumnado, y asume importantes cuotas de responsabilidad en las decisiones curriculares que se comparte".

Justamente en el contexto escolar se pueden ver manifestaciones que revelan estos tipos de profesores y las intenciones prioritarias contemporáneas buscan un profesor matizado con todas estas características, cuya expresión más acabada es el profesor como profesional. Carr y Kemmis (1988), analizan las profesiones desde tres criterios fundamentales:

1. "La existencia de un cuerpo de conocimientos provenientes de la investigación científica y la elaboración teórica. 
2. La asunción de un compromiso ético de la profesión, respecto a sus clientes.

3. El regirse por una normativa interna de autocontrol por parte del colectivo profesional".

El análisis de la profesión "la docencia" cumple con los criterios antes expresados, pero a condición de de que los profesionales de diferentes perfiles, convertidos en profesores universitarios, continúen su formación en aquellos contenidos relacionados con la preparación pedagógica que les posibilite:

?? Dirigir el proceso de enseñanza aprendizaje de la disciplina que desarrollan.

?? Investigar el propio proceso para su perfeccionamiento.

?? Incrementar su autonomía y control del propio trabajo.

?? Poseer un cuerpo de contenidos científicos consistentes y a su vez una ética compartida.

Según el Diccionario de la Real Academia la profesionalidad está definida como "la actividad de una persona que hace una cosa como profesión",. Por otra parte J. Añorga (1983) expresa que es "la cualidad de una persona que realiza su trabajo específico con relevante capacidad, sus objetivos, lo que se manifiesta en ejecutar las tareas con gran atención, exactitud y rapidez (...) sobre la base de una elevada preparación, incluyendo la experiencia."

Las universidades cubanas, seleccionan dentro de los mejores egresados de la Educación Superior, a aquellos que mayores posibilidades presentan para realizar funciones de profesor; pero en ese momento no tienen la madurez profesional ni la preparación para la docencia. Esta situación y la necesidad de formación pedagógica del profesorado universitario han sido el marco para el desarrollo de la tendencia denominada "profesionalización de la docencia".

La profesionalización de la docencia tiene como objetivo hacer de la docencia una actividad profesional, una profesión, una carrera; de tal forma que el ingeniero, el médico, el contador, el agrónomo, el arquitecto, independientemente de su formación inicial, puedan hacer de la docencia una actividad profesional.

Benedito (1991) reconoce en la profesionalización docente tres variables fundamentales:

\begin{tabular}{|l|l|l|}
\hline \multicolumn{1}{|c|}{ PREPARACION } & \multicolumn{1}{|c|}{ AUTONOMIA } & \multicolumn{1}{c|}{ AUTOCRITICA DE SERVICIO } \\
\hline $\begin{array}{l}\text {.Competencia y eficacia en la } \\
\text { actividad docente. }\end{array}$ & $\begin{array}{l}\text {.Un espacio laboral y social } \\
\text { propio. }\end{array}$ & .Prestigio profesional. \\
\hline Sentido artístico de la vida. & Control interprofesional. & $\begin{array}{l}\text {.Función de organización de la } \\
\text { cultura }\end{array}$ \\
\hline $\begin{array}{l}\text {.Un saber sistemático y } \\
\text { global (un saber profesional). }\end{array}$ & $\begin{array}{l}\text {.Aplicación a una entidad } \\
\text { colectiva profesional. }\end{array}$ & El trabajo en equipo. \\
\hline $\begin{array}{l}\text { Uso de un lenguaje técnico y } \\
\text { especifico. }\end{array}$ & $\begin{array}{l}\text {.Responsabilidad de una } \\
\text { tarea profesional. }\end{array}$ & \\
\hline $\begin{array}{l}\text { Participación en } \\
\text { investigaciones didácticas. }\end{array}$ & .La estabilidad laboral. & \\
\hline Formación continua. & .La capacidad de evaluar. & \\
\hline & La autocrítica profesional. & \\
\hline
\end{tabular}

El análisis empírico sobre la realidad escolar en varios lugares ha puesto de manifiesto que una gran parte de los profesores entienden como profesionalización: el progreso en la carrera docente. Sin embargo, existe una minoría que consideran la profesionalización vinculada a una perfección progresiva en su trabajo 
diario.

Los que mantienen la primera postura plantean que no cabe hablar de desarrollo profesional cuando uno comienza ejerciendo de maestro y se jubila realizando el mismo oficio. Ellos consideran que aunque existe cierto desarrollo este solo se hace realidad en la medida en que un profesor cambia de puesto para tener un mayor status social y que de no ser así esto impediría la carrera docente y el desarrollo profesional.

Se trata, en este caso, de un concepto elitista de profesionalización, basado en la distancia social respecto a los estudiantes y en el sentirse superiores, donde lo que más interesa es la promoción y no la cercanía y el intercambio con los estudiantes, estos generalmente asumen un intercambio frontal, unidireccional en el proceso de aprendizaje. Este concepto está poco acorde con las tendencias de la educación en la era moderna y con la forma en que la misma es llevada a la práctica.

Podría decirse quizás, que ellos ven el desarrollo profesional en su "fase terminal", cuando ya la experiencia acumulada le da cierta jerarquía al profesor.

Los que abrigan el segundo criterio se dan cuenta de que el profesional se caracteriza por la adquisición y aplicación de un conocimiento específico que se va convirtiendo en un compromiso permanente en su actualización y preparación para la vida de los estudiantes, generada por la reflexión en la acción, transformándose en un líder formal de grupo y utilizando la investigación como herramienta importante para el diagnóstico en el crecimiento de los grupos escolares, posee un gran sentido de pertenencia a su institución, a su enseñanza y a sus estudiantes.

\section{Modelos de formación del profesorado}

Se asume como definición de modelo la que parte de considerar que el modelo es un recurso para el desarrollo de la enseñanza, para la fundamentación científica de la misma, evitando que permanezca siendo una forma de hacer empírica y personal al margen de toda formalización científica.

Cada modelo es juzgado por su capacidad compresiva en cuanto a la realidad de la enseñanza, dado que cada uno de ellos posee esa propiedad de filtro que le es propia y develan diferentes aspectos de la misma.

La formación docente se concibe como el proceso permanente de adquisición, estructuración y reestructuración de conocimientos, habilidades y valores para el desempeño de la función docente.

La formación docente es continua, se lleva a cabo a lo largo de toda la práctica docente, tomándose dicha práctica como eje formativo estructurante.

Los modelos de formación del profesorado se han configurado históricamente sobre la base de dos concepciones: la primera define un conjunto de rasgos deseables en el profesional, y la segunda trasciende el ámbito de lo personal y visualiza al profesor en el contexto de la realidad compleja en la que se desempeña. Estos son:

?? El modelo teórico, cuyo propósito es formar profesionales capaces de responder a las exigencias que les plantee cualquier situación académica. 
?? El modelo crítico-reflexivo, que forma parte de un movimiento de renovación curricular y de la enseñanza màs amplia, que asume la idea, como eje central de este movimiento, de "profesor-investigador".

Es conveniente prescindir de un modelo de formación que integre ambas propuestas, contextualizandolo a la educación superior.

Zerchner (1983) establece cuatro paradigmas que a su juicio, es el punto de enmarcación teórica en la formación del profesorado:

1. El paradigma conductista, como formación del profesorado en competencias (comportamientos, conocimientos teóricos y prácticos). Su objeto es ofrecer a los alumnos capacidades docentes para decidir lo que tienen que hacer en situaciones reales. El criterio fundamental de la eficiencia docente es el desarrollo de aptitudes y conocimientos de los alumnos, medidos antes y después de la influencia del profesor.

2. El paradigma personalista o $\underline{\text { humanista, }}$, regido por el criterio de que la enseñanza es básicamente un proceso de relación interpersonal y desarrollo personal. Un "buen profesor es como un artista habilidoso capaz de facilitar el desarrollo personal del alumno".

3. El paradigma tradicional-artesanal, la enseñanza es un proceso de ensayo-error, donde se aprende mejor al pasar un período de prácticas. Entiende al profesor como un transmisor de contenidos culturales, razón por la que también se denomina paradigma culturalista o racionalista. Este paradigma ve el aprendizaje de la enseñanza como un proceso de modelado en que el alumno aprendiz imita al maestro.

4. El paradigma crìtico-reflexivo, orientado a la indagación centrada en la investigación critica y en la reflexión de las causas y consecuencias de las acciones en la clase. Trata de dar a los profesores la capacidad intelectual de valorar su propia práctica, así como establecer una conexión entre la formación del profesor y la realidad sociopolítica exterior, con la aspiración de mejorar el mundo a través de la educación.

Al efectuar el análisis de los paradigmas anteriores, se infiere, la presencia de los mismos en diferentes modelos de formación del profesorado, no obstante en la actualidad existe una tendencia marcada hacia un mayor empleo del crítico reflexivo al acentuar la importancia de las potencialidades que reviste un proceso de formación concientizador, intelectual y analítica.

\section{Modelos de formación permanente del profesorado}

La educación permanente puede definirse como el proceso que mejora los conocimientos referentes a la actuación, las estrategias y las actitudes de quienes trabajan en las instituciones educativas. La finalidad prioritaria de la formación permanente es favorecer el aprendizaje de los estudiantes a través de la mejora de la actuación del profesorado.

Siguiendo el estudio elaborado por Spark y Loucks-Horsley (1990) en lo referente a la formación permanente, pueden agruparse en cinco modelos que sirven de punto de referencia, pero que no pueden 
considerarse cerrados en sí mismos. Pueden encontrarse en ellos estrategias, y actitudes comunes, e incluso la finalidad puede ser la misma (producir una mejora en el aprendizaje de los estudiantes o en la gestión de la institución, a partir de la formación del profesorado), pero lo que cambia son las concepciones, las actitudes frente a la formación y la enseñanza.

Por esta razón, ante cada uno de estos modelos, para la formación permanente de los docentes, es posible preguntarse desde una posición reflexiva: ¿en qué medida este modelo afectará a la actuación del profesor en el aula? ¿por qué este modelo debería ser adoptado? ¿qué indica que este modelo producirá diferencias notables en la actuación de los docentes?.

Responder a estas preguntas supone no sólo acercarse a la definición de cada uno de estos modelos sino, además, conocer su fundamentación tanto teórica como en la investigación, las fases en las que puede realizarse, así como valorar sus posibilidades de aplicación.

\section{Formación orientada individualmente}

Este modelo se caracteriza por ser un proceso en el cual los mismos maestros y profesores son los que planifican y siguen las actividades de formación que creen puedan facilitar su aprendizaje.

La fundamentación de este modelo parte de una observación de sentido común. Los docentes aprenden muchas cosas por sí mismos, mediante la lectura, la conversación con los colegas, la puesta a prueba de nuevas estrategias de enseñanza, la confrontación reflexiva con su propia práctica diaria, la propia experiencia personal, etc.

En todas estas situaciones los docentes aprenden sin la presencia de un programa formal y organizado de formación permanente. Partiendo de esta evidencia es posible planificar la formación del profesorado mediante programas que promuevan actividades que faciliten el aprendizaje individualizado.

La característica principal de este modelo es que el aprendizaje lo diseña el propio maestro o profesor. Es él quien determina sus propios objetivos y selecciona las actividades de formación que pueden ayudarle a cubrir tales objetivos.

Los docentes pueden aprender individualmente realizando una investigación, llevando a cabo un proyecto de innovación subvencionado, realizando materiales curriculares $u$ otras actividades relacionadas con el trabajo profesional de los enseñantes.

El modelo "Formación orientada individualmente" contempla muchos aspectos que son positivos, no obstante la ausencia de un "programa organizado" debilita las posibilidades que ofrece el modelo, ya que es posible lograr un programa donde se parta de las necesidades individuales, y los docentes participen en la elaboración del programa y los objetivos que persiguen.

\section{El modelo de obsevación-evaluación}

Este modelo se caracteriza por dirigirse a responder a la necesidad del docente, de saber cómo está afrontando la práctica diaria para aprender de ella. 
Otra premisa que subyace en este modelo es que la reflexión individual sobre la propia práctica puede mejorar con la observación de otros, la discusión y la experiencia en común.

Este modelo está asociado a una evaluación, según el criterio de muchos de los docentes, por lo que en general no lo consideran como una ayuda y tienen dificultades para entender sus ventajas. Tradicionalmente los docentes consideran a su clase como un lugar privado al que sólo se accede desde una posición de autoridad (el inspector para evaluarlo o el investigador para obtener datos), lo que limita en gran medida la efectividad que pueda tener el modelo.

\section{El modelo de desarrollo y mejora de la enseñanza}

Este modelo tiene lugar cuando los profesores están implicados en tareas de desarrollo curricular, diseño de programas, o en general mejora de la institución y tratan con todo ello de resolver problemas generales o específicos relacionados con la enseñanza.

Este modelo de formación supone, una combinación de modos y estrategias de aprendizaje que resulta de la implicación de los docentes en tal proceso.

La fundamentación de este modelo está en la concepción de que los adultos aprenden de manera más eficaz cuando tienen necesidad de conocer algo concreto o han de resolver un problema. Esto hace que en cada situación el aprendizaje de los profesores se guíe por la necesidad de dar respuestas a determinados problemas.

Otra idea que apoya este modelo es que las personas que están próximas a su trabajo tienen una mejor comprensión de lo que se requiere para mejorarlo.

El modelo de desarrollo y mejora de la enseñanza supone una combinación de modos y estrategias de aprendizaje que resulta de la implicación de los docentes en tal proceso, no obstante, es insuficiente la actuación que presta al impacto de las experiencias de los profesores, así como a la mejora profesional a la que les ha llevado este proceso.

\section{El modelo de entrenamiento}

En este modelo, los objetivos, el contenido y el programa lo establecen la administración o los formadores, aunque hay algunas propuestas que implican a los participantes en la planificación inicial del programa.

La concepción básica que apoya este modelo es que hay una serie de comportamientos y técnicas que merecen que los profesores la reproduzcan en clase.

Otra referencia que fundamenta este modelo es que los profesores pueden cambiar su manera de actuar y aprender a reproducir comportamientos en sus clases que no tenían previamente. Por esta razón se estima que este modelo constituye un medio para adquirir conocimientos y estrategias de actuación.

La teoría y la investigación sobre este modelo proceden de diferentes fuentes, en las que se refleja que, según los resultados esperados, el entrenamiento puede incluir exploración de la teoría, demostración de 
estrategias, prácticas de las mismas en situación de simulación, devolución sobre la actuación y asesoría en el lugar de trabajo.

Este modelo cubre en buena medida los objetivos que se esperan si se realizan en todas sus fases y que los docentes pueden mostrar cambios significativos en sus conocimientos y actuaciones en el aula.

\section{El modelo indagativo o de investigación}

Este modelo requiere que el profesorado identifique un área de interés, recoja información y basándose en la interpretación de estos datos, realice los cambios necesarios en la enseñanza.

Puede ser una actividad individual o hecha en grupos pequeños o llevada a cabo por todo el claustro de una institución. Es un proceso que puede ser formal o nformal, y puede tener lugar en la clase, en un departamento docente de profesores, o puede ser el resultado de un curso en la universidad.

Los profesores desarrollan nuevas formas de comprensión cuando ellos mismos contribuyen a formular sus propias preguntas y recogen sus propios datos para darles respuestas.

Las bases de este modelo se encuentra en las propuestas de Dewey, quien escribió que los profesores necesitan "una acción reflexiva". Uno de los impulsores actuales de este modelo es Zeichner quien señala que desde hace mas de 30 años se ha ido planteando el tema de "los profesores como investigadores sobre la acción", "Ios profesores como innovadores", "los profesores que se autodirigen" y "los profesores como observadores participante".

Uno de los elementos más importantes que fundamenta este modelo es que la investigación es importante para el profesorado, pues por medio de ella detecta y resuelve problemas y, en este contexto, pueden crecer como individuos.

Cuando los docentes actúan como investigadores, el resultado es que toman decisiones al estar mejores informados, sus experiencias les sirven de apoyo para una mayor colaboración entre ellos y aprenden a ser mejores profesores, siendo capaces de observar más allá de lo inmediato, de lo individual y de lo concreto.

Pero la principal aportación de este modelo es que cuando la administración, los formadores y los docentes trabajan juntos, cada uno puede aprender de la perspectiva del otro y buscar soluciones.

Se considera que los modelos valorados son portadores de aspectos importantes a tener en cuenta en la elaboración de un proyecto de formación permanente que contribuya significativamente a la profesionalización de los docentes; por lo que un tipo de combinación entre ellos se hace necesario en $d$ contexto universitario.

\section{Propuesta para la formación pedagógica del profesorado universitario}

Se concibe la formación pedagógica del profesorado universitario como un proceso continuo que atendiendo a diferentes etapas organizadas en su práctica docente, facilitan iniciar, adiestrar, formar y perfeccionar a dichos profesores en el dominio de los contenidos de la didáctica de la educación superior con el 
propósito de incidir en la calidad de la formación de los estudiantes lo que influye en la calidad de la educación superior.

A continuación se detallan las diferentes etapas que pueden ser organizadas durante los procesos de formación pedagógica:

\section{Iniciación docente}

El profesor novel que se inserta a realizar las funciones docente solo posee formación cient ífica sobre la disciplina que explica, pero no dispone de los fundamentos pedagógicos para dirigir el proceso de enseñanza aprendizaje. Durante esta etapa se familiariza con el estudio de los documentos normativos de la educación superior, participa en la supervisión de actividades realizadas por profesores de experiencia, discutiendo con ellos los resultados de la observación; similar estrategia se desarrolla con los profesores noveles al ser visitados. Todas las actividades mencionadas son planificadas por el departamento docente al cual pertenece el profesor.

\section{Adiestramiento docente}

Esta etapa, fundamentalmente, se realiza a través del trabajo metodológico en los diferentes niveles organizativos en que está implicado el profesor. Durante dicha etapa el profesor participa de forma activa en las diferentes actividades metodológicas concebidas por su departamento docente, siendo protagonista en la realización de clases abiertas, disertaciones de trabajos pedagógicos, visitas a profesores de experiencia con el propósito de mejorar la calidad de sus clases.

\section{Formación pedagógica por niveles}

A partir de un diagnóstico de necesidades de aprendizaje realizado, a los profesores, éstos son ubicados en los distintos niveles de formación y consecuentemente, se estructuran en sistemas los diferentes postgrados a cursar. Para ello se toma en consideración las necesidades personales, sociales e institucionales.

Los niveles de formación pedagógica previstos son:

Básico: Incluye a los profesores que no han cursado estudios sobre la Didáctica de la Educación Superior. Mediante el curso básico de Pedagogía los profesores recibirán los conocimientos y habilidades esenciales sobre el desarrollo del proceso de enseñanza aprendizaje de la Educación Superior.

Básico actualizado: Comprende la actualización de los profesores en los componentes del proceso de enseñanza aprendizaje superior y su aplicación práctica a los diferentes niveles organizativos. Los profesores se agrupan en un curso de actualización pedagógica.

Profundización: Está concebido para los jefes de carrera, departamento, colectivo de años, disciplina y asignatura, así como para otros profesores y directivos que lo requieran. Estos profesores participan en el diplomado de didáctica y dirección de la Educación Superior. 
Especialización. Esta destinado a la especialización en los contenidos de las Ciencias de la Educación vinculado a las necesidades del puesto laboral. Los profesores designados participan en cursos de especialización diseñados a tales efectos.

4. Formación académica investigativa. Está dirigida al perfeccionamiento continuo del profesor universitario, dicha formación se da a lo largo de su vida profesional. Incluye actividades de autosuperación que realiza el profesor para sistematizar y profundizar los conocimientos obtenidos a través del sistema de formación pedagógica acreditado por la Universidad. Asimismo se incorpora a todas aquellas actividades organizadas por su institución u otras para continuar elevando su desarrollo profesional y personal. Se ofrecen como alternativas para continuar su formación los programas de Maestría en Educación y Doctorados en Ciencias Pedagógicas y en Educación.

En cada uno de los niveles se brinda especial atención a la participación en eventos pedagógicos para potenciar el intercambio académico con otros profesores y contraponer sus ideas, creencias, opiniones sobre el perfeccionamiento de la labor docente de la Educación Superior.

Las cuatro etapas referidas están íntimamente vinculadas y van proyectando el trabajo de investigación de los profesores alrededor de su propio proceso.

Desde esta perspectiva, necesariamente el profesor para lograr una formación pedagógica, según las exigencias de la Educación Superior actual, no tiene que transitar por cada una de las 3 primeras etapas señaladas, esto estará en dependencia del nivel de desarrollo profesional y pedagógico que ha alcanzado a través de su práctica docente.

La propuesta de formación pedagógica se caracteriza por:

?? El uso del diagnóstico pedagógico

Se parte de la identificación de las necesidades de aprendizajes personales, sociales e institucionales para organizar el sistema de cursos que se ofertan.

?? El carácter participativo de los profesores

Los profesores participan en la elaboración de los programas que se ofertan con vistas a responder a las necesidades de aprendizajes señaladas.

?? El empleo del sistema de principios didácticos de la educación superior

Los programas de los cursos que se desarrollan tienen en cuenta los principios de carácter científico de la enseñanza, vincular la teoría con la práctica, sistematización, atender a las diferencias individuales, asimilación, accesibilidad.

?? La integración del trabajo individual al de grupo

Los programas de los postgrados están concebidos para el trabajo en grupo en sus diferentes modalidades, con ello se garantiza reflexionar de manera individual y colectiva sobre la práctica docente diaria. 
?? El intercambio permanente de experiencias entre los profesores

Las diferentes etapas en que puede organizarse la formación pedagógica tributan al intercambio permanente de experiencias entre los profesores lo cual propicia apropiarse de modos de actuación docentes.

?? El mantener una retroalimentación constante

Se recoge sistemáticamente información, a partir del empleo de diferentes técnicas y procedimientos, sobre la marcha del desarrollo del curso impartido.

?? La personificación de la profesión

Implica la incorporación a los profesores universitarios de los modos de actuación propios de la Educación Superior.

?? El impacto del programa impartido

Los resultados de la preparación pedagógica que van recibiendo los profesores se revierten en los aprendizajes de los estudiantes a partir del perfeccionamiento del proceso de enseñanza aprendizaje.

La característica esencial de esta propuesta es la satisfacción de las necesidades de aprendizaje personales, sociales e institucionales a partir de la estructuración de programas formales en diferentes modalidades los cuales están dirigidos a la formación pedagógica de los profesores universitarios y en última instancia a mejorar la calidad de la formación del estudiante.

La fundamentación teórica de la propuesta se basa en el enfoque histórico cultural de Vigosky, parte de la consideración de que el aprendizaje es un proceso social muy vinculado al desarrollo del sujeto. En ella se ponen de manifiesto las siguientes relaciones:

?? Relación entre personalidad y actividad.

?? Relación entre aprendizaje y desarrollo.

?? Relación entre lo individual y los social.

La propuesta para la formación del profesorado universitario, a la luz de las nuevas tecnologías implica:

?? Prestar atención a la diversidad en el proceso evaluativo.

?? Modificar el énfasis tradicionalmente puesto en los procesos de enseñanza por uno centrado en el aprendizaje

?? Favorecer mayor protagonismo de los profesores en su propio aprendizaje.

?? Pasar de un modelo de enseñanza uniforme para toda la clase al trabajo en pequeños grupos.

?? Incentivar el estímulo al aprendizaje permanente. 
?? Posibilitar el desarrollo de competencias de toma de decisiones ante propuestas alternativas.

?? Emplear las NTIC.

\section{CONCLUSIONES}

?? Cualquiera que sea la propuesta de formación pedagógica del profesorado universitario adoptado, debe estar orientada a elevar la calidad de la educación.

?? Una propuesta para la formación pedagógica de los profesores universitarios que se conciba como un proceso continuo, integrador, holístico puede estar organizado en las siguientes etapas:

- iniciación docente

- adiestramiento docente.

- formación pedagógica por niveles.

Formación académica investigativa.

?? La propuesta de formación pedagógica de los profesores universitarios diseñada se caracteriza por: el uso del diagnóstico pedagógico, el carácter participativo de los profesores, el empleo del sistema de principios didácticos de la Educación Superior, la integración del trabajo individual al de grupo, el intercambio permanente de experiencias, una retroalimentación constante, la personificación de la profesión y el impacto del programa impartido.

?? La formación pedagógica del profesorado universitario cobra en la actualidad una significativa importancia a los efectos de estimular la innovación, el sentido crítico, la reflexión, la creatividad en función de cubrir con las necesidades de aprendizaje que demanda su práctica docente, todo ello contribuye a elevar la calidad de la formación del estudiante de la Educación Superior.

\section{RECOMENDACIONES}

Favorecer la integración en redes, entre las universidades, de propuestas de formación pedagógica del profesorado universitario.

\section{BIBLIOGRAFÍA}

AGUIRRE CÁRDENAS, JESÚS. Formación pedagógica y didácticas universitarias. http//www.nhc.noaa.gov/ftp/graphiscs/ATB/AL 1302 W.GIF. 6 p.

BARRIOS, R. OSCAR (2001). La formación docente: Teoría y práctica. Centro de Informaciones pedagógicas. Universidad Metropolitana de Ciencias de la Educación. 6 p.

CASCANTE, C. (1996). Proyecto docente de Didáctica General. Universidad de Oviedo. Inédito. 
CARR, W; KEMMIS, S. (1988). Teoría crítica de la enseñanza. La investigación-acción en la formación del profesorado. Editora Martínez Roca. Barcelona.

COLECTIVO DE AUTORES. MINED. (1998) Documentos normativos para el perfeccionamiento del Sistema Nacional de Educación. Editorial Pueblo y Educación. Ciudad de La Habana.

DEL CARMEN, L. (1990). Desarrollo curricular y formación permanente del profesorado. En Gil Pérez. Formación de formadores en Didáctica de las Ciencias. Editorial Nau Llibres. Valencia. 1990 p $45-$ 48

DE MIGUEL, M. Y OTROS. (1996). El desarrollo profesional docente y las resistencias a la innovación educativa. Servicio de publicaciones de la Universidad de Oviedo. Oviedo.

DE LELLA, CAYETANO. (1999). Modelos y tendencias de la formación docente. I Seminario Taller sobre perfil del docente y estrategias de formación. Lima, Perú. 1999. 9 p.

ESCONTRELA MAO, RAMÓN. (1992). La formación del profesor, modelos y tendencias: el modelo crítico reflexivo. Revista de Pedagogía. (Venezuela), (29). Enero Marzo, 1992. 63-81 p.

ENCICLOPEDIA GENERAL DE LA EDUCACIÓN. (2000) Tomo I. Oceano Grupo Editorial. Barcelona. FARIÑAS, G. (1990). La selección de tareas docentes en el proceso de dirección de la enseñanza superior. Universidad de La Habana, La Habana. Inédito.

FERNÁNDEZ PÉREZ, M. (1988). La profesionalización docente en la escuela. Escuela Español. S.A. Madrid.

GARCÍA CRUZ, RUBÉN. (1999). La profesionalización docente en la escuela. Tesis presentada en opción al título Académico de Máster en Ciencias Pedagógicas. ISP Conrado Benítez. Cienfuegos, 1999. $68 \mathrm{p}$.

GARCÍA GARRIDO, J.L.(1998). El futuro de la universidad en Europa y en España. Conferencia pronunciada en la Universitat d'Estiu de la Universidad Rovira i Virgili en julio de 1998. Tarragona.

GIMENO, SACRISTÁN, J. Y PÉREZ GÓMEZ, A. (1982). La enseñanza: su teoría y su práctica. Madrid: Akal universitaria. 1982. $479 \mathrm{p}$.

GIROUX, H. A. (1990) Los profesores como intelectuales. Ediciones Paidós/MEC. Barcelona.

GONZÁLEZ, OTMARA. (1991). El enfoque histórico cultural como fundamento de una concepción pedagógica. Tendencias Pedagógicas Contemporáneas. Colectivo de autores. Universidad de La Habana. CEPES. Ciudad de La Habana 1991. 92-114 p.

IGLESIAS LEÓN, MIRIAM Y OTROS. (2002). La preparación pedagógica de los profesores universitarios. Su impacto en la calidad de la Educación Superior. Ponencia presentada en $3^{\text {a }}$ Convención Internacional de la Educación Superior. Universidad 2002. 12 p.

IMBERÓN, F. (2000) La formación y el desarrollo profesional del profesorado universitario. Hacia una nueva cultura profesional. Editorial Graó. Barcelona. 
MOTA ENCISO, FLAVIO, Reflexiones sobre educación. La docencia como actividad profesional. http://www.uag.mx/83/egresados.html.

NOGUERA ARROM, JOANA. (2001) La formación pedagógica del profesorado universitario. Revista Bordón 53 (2), 2001. 269-277 p.

PÉREZ GÓMEZ, A. (1993) La formación del docente como intelectual comprometido. Revista Signos. 8/9 1993. $42-53 \mathrm{p}$.

PRIMO FERNÁNDEZ, MANUEL. (2001). Modelo básico para la superación del docente como investigador. Tesis de Maestría. Universidad de Cienfuegos.

RODRÍGUEZ, JOSÉ Ma. (1995). Formación de profesores y prácticas de enseñanza. Un estudio de caso. Universidad de Huelva. Publicaciones. España. 1995. 229 p.

UNESCO. Conferencia mundial sobre la educación superior. La educación superior en el XXI: visión y acción. Bruselas: UE.

\section{Referencias bibliográficas}

1- Declaración mundial sobre la educación superior en el siglo XXI: visión y acción, art. 9, apart, d, p 5.

2- Marco de acción prioritaria para el cambio y el desarrollo de la educación superior. UNESCO. Punto 1.6 d; p. 51998. 


\title{
Contactar
}

Revista lberoamericana de Educación

\author{
Principal OEI
}

